\title{
A new Dolbeault complex in quaternionic and Clifford analysis
}

\author{
Fabrizio Colombo \\ Dipartimento di Matematica \\ Politecnico di Milano \\ Via Bonardi, 9 \\ 20133 Milano, Italy \\ fabcol@mate.polimi.it \\ Irene Sabadini \\ Dipartimento di Matematica \\ Politecnico di Milano \\ Via Bonardi, 9 \\ 20133 Milano, Italy \\ sabadini@mate.polimi.it
}

\author{
Alberto Damiano \\ Department of Mathematical Sciences \\ George Mason University \\ Fairfax, VA 22030 USA \\ adamiano@gmu.edu \\ Daniele C. Struppa \\ Department of Mathematical Sciences \\ George Mason University \\ Fairfax, VA 22030 USA \\ dstruppa@gmu.edu
}

\begin{abstract}
The papers introduces a new complex of differential forms which provides a fine resolution for the sheaf of regular functions in two quaternionic variables and the sheaf of monogenic functions in two vector variables. The paper announces some applications of this complex to the construction of sheaves of quaternionic and Clifford hyperfunctions as equivalence classes of such differential forms.
\end{abstract}

\section{Introduction}

In this paper we present some new ideas on complexes of differential forms in the quaternionic and the Clifford settings. In particular we announce some new results and some research directions, which are particularly promising and are giving a new insight in the possibility of constructing a theory of multivariable quaternionic and Clifford valued hyperfunctions.

The study of the Cauchy-Fueter systems for functions of a quaternionic variable dates back to the twenties, when Fueter introduced the system as a way to generalize holomorphicity to the case of functions defined on the space of quaternions. The theory for the case of a single quaternionic variable is quite well known (and the solutions of the Cauchy-Fueter system are known as regular functions), while the case of several quaternionic variables (and correspondingly several CauchyFueter systems) has only recently been understood, and the most up-to-date description is given in [3]. More recently, the field of Clifford analysis has developed as the study of solutions of the Dirac equation (or system) for Clifford valued functions (these functions are usually referred to as monogenic functions). Once again, one finds properties which generalize holomorphicity, and even though the theory is by now very well developed, see for example [1], and [4], most of the results are restricted to the case of a single Dirac equation. The only attempt to extend the study to the case of several Dirac equations is brought forward in [3], but the results are far from being complete. 
Given the many formal similarities between the theory of regular functions of quaternionic variables, the theory of monogenic functions, and the classical theory of holomorphic functions, it is no surprise that several attempts have been made towards the creation of a theory of quaternionic and Clifford hyperfunctions to be interpreted as suitable boundary values of regular and monogenic functions. The only known work in the case of regular functions is due to the authors (see the references in [3]), while the most important work for the Clifford case is due to Sommen (see [6] and references therein). The state of the art on this problem is given in the recent monograph [3].

All the existing work, however, only deals with rather simple extensions of the theory of onedimensional hyperfunctions. In other words, the existing literature always consider solutions to a single differential equation (though of course both the Cauchy-Fueter and the Dirac equation can be thought of as systems). Because of the way in which the Cauchy-Fueter and the Dirac operators are defined, and since the theory of regular functions in one quaternionic variable and the theory of monogenic functions are essentially uni-dimensional theories, the corresponding hyperfunction and microfunction theories always share the flavor of the standard theory of hyperfunctions in one variable.

Until recently, however, very little progress had been done in the attempt to find a way to develop a hyperfunction theory for boundary values of regular and monogenic functions in several quaternionic or Clifford variables. A significant obstacle to progress in this direction was the lack of an appropriate theory of differential forms which could fulfill the role that the Dolbeault theory plays in the complex case. In the last couple of years, there was finally some advance with the introduction, by F. Sommen, of the notion of megaforms and with the understanding of their connection with our analysis of multidimensional Dirac systems [5].

In this paper we announce several important results on megaforms for regular functions of several quaternionic variables and for multivariable monogenic functions. Specifically, in sections two and three, we show that it is possible to construct a Dolbeault-like complex, which provides a fine resolution for the sheaves of regular and monogenic functions in two variables. In section four we point out the many important consequences of our construction and we discuss how this will lead to a full hyperfunction theory for multivariable regular and monogenic functions. The detailed proofs will appear elsewhere ([2]).

Acknowledgements. The authors are grateful to the organizers of ISAAC 2005 for inviting them to speak at the conference. The first three authors are grateful to George Mason University for its financial support.

\section{Clifford megaforms for the bi-dimensional Dirac system}

Let $\mathbb{R}^{m}$ be the real Euclidean space: we define $\mathbb{R}_{m}$ as the real Clifford algebra generated by the basis elements $\left\{e_{1}, \ldots, e_{m}\right\}$ together with the defining relations

$$
e_{j} e_{k}+e_{k} e_{j}=-2 \delta_{j k} .
$$

Let $x_{1}, \ldots, x_{m}$ denote the standard (commuting) variables, $\underline{x}=\sum_{j=1}^{m} x_{j} e_{j}$ and let $f(\underline{x})$ be an $\mathbb{R}_{m}$-valued function. One may consider the action of the Dirac operator, or vector derivative,

$$
\partial_{\underline{x}}: f(\underline{x}) \rightarrow \partial_{\underline{x}} f(\underline{x})=\sum_{j=1}^{m} e_{j} \partial_{x_{j}} f(\underline{x})
$$

and the solutions of the homogeneous equation $\partial_{\underline{x}} f(\underline{x})=0$, that are called monogenic functions. 
Consider now several vector variables

$$
\underline{x}_{1}, \ldots, \underline{x}_{n}, \quad \underline{x}_{j}=\sum_{k=1}^{m} x_{j k} e_{k},
$$

and functions $f\left(\underline{x}_{1}, \ldots, \underline{x}_{n}\right)$ with values in $\mathbb{R}_{m}$. We then define monogenic functions in $n$ copies of $\mathbb{R}^{m}$ to be those functions which satisfy the following multivariable Dirac system:

$$
\left\{\begin{array}{c}
\partial_{\underline{x}_{1}} f=0 \\
\cdots \\
\partial_{\underline{x}_{n}} f=0
\end{array}\right.
$$

The final piece of notation we want to introduce is the notion of radial algebra.

Definition 2.1. Let $S$ be a set of objects which we will consider as "abstract vector variables". The radial algebra $R(S)$ is defined to be the associative algebra generated by $S$ over $\mathbb{R}$ with the defining relations

$$
[\{x, y\}, z]=x y z+y x z-z x y-z y x=0, \text { for } x, y, z \in S .
$$

Note that such an algebra could be constructed over any suitable ring, though in this paper we will confine ourselves to $\mathbb{R}$. Let $T(S)$ be the tensor algebra generated by the elements of $S$, and let $I(S)$ be the two-sided ideal generated by the polynomials

$$
[\{x, y\}, z] \text {. }
$$

Then

$$
R(S)=T(S) / I(S)
$$

The notion of radial algebra is particularly useful, because it turns out that Dirac operators in several variables satisfy exactly the relations which define the radial algebra, and nothing more.

This section is devoted to a recap of some of the main results from [5], with special interest for the case of two variables (this means that our monogenic functions are defined on $\left(\mathbb{R}^{m}\right)^{2}$, and have values in $\mathbb{R}_{m}$ ). The ideas contained in this section are the inspiration for section three.

We begin by introducing what we have called megaforms as the analogue of classical differential forms for real coordinates. To define the classical basic differential forms one starts from the operator $d=\sum \partial_{x_{j}} d x_{j}$ acting on the algebra generated by $x_{1}, \ldots, x_{m}, d x_{1}, \ldots, d x_{m}$ together with the properties

1. $d(\omega)=d\left(\sum F_{j} d g_{j}\right)=\sum d F_{j} \wedge d g_{j}$,

2. $d(f \omega)=d f \wedge \omega+f d \omega$,

3. $d x_{j} \wedge d x_{k}=-d x_{k} \wedge d x_{j}$.

If one replaces $x_{1}, \ldots, x_{m}$ with the generators of a radial algebra, which we will denote in the same way, it is natural to replace the partial derivatives with the vector derivatives $\partial_{x_{1}}, \ldots, \partial_{x_{n}}$, but it is not so clear how to generalize the properties above. Instead, we try to generalize the formula " $d=\sum \partial_{x_{j}} d_{x_{j}}$ " in the case of vector derivatives $\partial_{x_{1}}, \ldots, \partial_{x_{n}}$, which themselves satisfy the defining relations for a radial algebra. To be able to make use of the radial algebra defining relations, we will construct spaces $F_{k}$ of forms, and differentials $d^{k}: F_{k} \rightarrow F_{k+1}$ such that $d^{k+1} d^{k}=0$. 
However, we will recognize that the differentials $d^{k}$ may consist of two pieces: a "degree one" piece $d_{1}^{k}$ of the form $\sum_{j=1}^{m} D_{j}^{k} \partial_{j}$ and a "degree two" piece $d_{2}^{k}$ of the form $\sum_{i, j=1}^{m} D_{i j}^{k} \partial_{i} \partial_{j}$. The symbols of degree one, $D_{j}^{k}$, are the Dirac analogue of the $d x_{j}$ which are used in the classical de Rham complex, while the symbols of degree two, $D_{i j}^{k}$, are new symbols which will be necessary to reflect the existence of quadratic syzygies. As such, these symbols will have to satisfy some axioms in order to guarantee $d^{k+1} d^{k}=0$. Note also that the symbol $D_{j}^{k}$ and the symbols $D_{i j}^{k}$ may, a priori, depend on $k$, though we will see that the full theory can be developed with only a minimal dependence on $k$.

Definition 2.2. Let $x_{1}, \ldots, x_{n} \in S$; the algebra $M\left(x_{1}, \ldots, x_{n} ; S\right)$ of free megaforms in the variables $x_{1}, \ldots, x_{n}$ with coefficients in $R(S)$ is the associative algebra which is generated over the algebra $R(S)$ by the set of "basic megaforms" $\left\{D_{i}^{k}, D_{j \ell}^{k}: i, j, \ell=1, \ldots, n\right\}$ together with the identities derived from $d^{k+1} d^{k}=0$.

To our purposes, the position of the various symbols $D_{i}^{k}, D_{j \ell}^{k}$ in the relations we obtain makes clear that we can omit the top label. It suffices to keep in mind that the relations obtained for $D_{j \ell}=D_{j \ell}^{k}$ at the $k$-th step cannot be used for $D_{j \ell}=D_{j \ell}^{k+1}$. From now on we will write $D_{i}, D_{j \ell}$, and we will leave it to the reader to distinguish among the different levels (in fact the order in which they appear identifies their level immediately).

We are now ready to discuss directly the case of systems of two Dirac operators.

Let $\mathcal{M}$ be the space of monogenic functions in two variables $x_{1}, x_{2}$. Let $F_{0}$ be the space of $\mathcal{C}^{\infty}$ functions in $x_{1}, x_{2}$ and let $F_{1}$ be the space of "1-forms" whose elements are written as $g=\sum_{i=1}^{2} D_{i} g_{i}, g_{i} \in F_{0}$, so that if $d^{0}=\sum_{i=1}^{2} D_{i} \partial_{i}$, where $\partial_{i}=\partial_{x_{i}}$, we have an exact sequence

$$
0 \rightarrow \mathcal{M} \hookrightarrow F_{0} \stackrel{d^{0}}{\rightarrow} F_{1}
$$

The next step in the construction of the complex consists in defining a space $F_{2}$ of "2-forms" and a suitable "differential" $d^{1}: F_{1} \rightarrow F_{2}$ such that $d^{1} d^{0}=0$. As we explained above, we postulate that $d^{1}$ be made of two components $d_{1}^{1}$ and $d_{2}^{1}$ of degrees, respectively, one and two. Thus we assume, for $g \in F_{1}$,

$$
d^{1} g=d_{1}^{1} g+d_{2}^{1} g=\sum_{j, k=1}^{2} D_{k} D_{j} \partial_{k} g_{j}+\sum_{i, j, k=1}^{2} D_{k i} D_{j} \partial_{k} \partial_{i} g_{j}
$$

The condition $d^{1} d^{0}=0$ implies that $\sum_{j, k=1}^{2} D_{k} D_{j} \partial_{k} \partial_{j} f=0$, i.e., that for any $k$ and $j, D_{k} D_{j}=0$. But then, because of the form of elements in $F_{1}$, one has that $d_{1}^{1} \equiv 0$, and therefore

$$
d^{1} g=d_{2}^{1} g=\sum_{i, j, k=1}^{2} D_{k i} D_{j} \partial_{k} \partial_{i} g_{j}
$$

Proposition 2.3. Let $d^{0}=D_{1} \partial_{1}+D_{2} \partial_{2}$ and $d^{1}=D_{11} \partial_{1}^{2}+D_{12} \partial_{1} \partial_{2}+D_{21} \partial_{2} \partial_{1}+D_{22} \partial_{2}^{2}$. Then $d^{1} d^{0}=0$ implies the following:

$$
D_{i j} D_{i}=0 \quad i, j=1,2 \quad D_{i i} D_{j}+D_{j i} D_{i}=0 \quad i, j=1,2, \quad i \neq j .
$$

Remark 2.4. The relations (3) are the analogues of the complex relations $d \bar{z}_{1} \wedge d \bar{z}_{2}=-d \bar{z}_{2} \wedge d \bar{z}_{1}$, $d \bar{z}_{i} \wedge d \bar{z}_{i}=0$. 
We now study the kernel of the map $d^{1}: F_{1} \rightarrow F_{2}$ and we note that in [5] we have shown that a 1 -form $g$ is $d^{1}$-closed if and only if its components $g_{j}$ satisfy the compatibility conditions of the system $d^{0} f=g$.

Proposition 2.5. Let $g=D_{1} g_{1}+D_{2} g_{2}$ be an element of $F_{1}$. Then $d^{1} g=0$ if and only if

$$
\partial_{i}^{2} g_{j}-\partial_{j} \partial_{i} g_{i}=0, \quad i=1,2
$$

i.e. $d^{1} g=0$ if and only if $\left(g_{1}, g_{2}\right)$ satisfy the compatibility conditions for the solvability of the system

$$
\left\{\begin{array}{c}
\partial_{1} f=g_{1} \\
\partial_{2} f=g_{2}
\end{array}\right.
$$

We know, from the general theory, that the complex closes with one more linear condition that is the compatibility condition for the solvability of the system

$$
\left\{\begin{array}{c}
\partial_{1}^{2} g_{2}-\partial_{2} \partial_{1} g_{1}=h_{12} \\
\partial_{2}^{2} g_{1}-\partial_{1} \partial_{2} g_{2}=h_{21}
\end{array}\right.
$$

Interestingly enough this condition can be derived using megaforms and their closure.

Proposition 2.6. Let $d^{1}=D_{11} \partial_{1}^{2}+D_{12} \partial_{1} \partial_{2} 2+D_{21} \partial_{2} \partial_{1}+D_{22} \partial_{2}^{2}$ and let $d^{2}=D_{1} \partial_{1}+D_{2} \partial_{2}+$ $D_{11} \partial_{1}^{2}+D_{12} \partial_{1} \partial_{2} 2+D_{21} \partial_{2} \partial_{1}+D_{22} \partial_{2}^{2}$ then $d^{2} d^{1}=0$ implies for $i, j=1,2, i \neq j$

$$
D_{i i}^{2} D_{j}=0, \quad D_{i i} D_{j j} D_{i}-D_{i j} D_{i i} D_{j}=0, \quad D_{i j} D_{j j} D_{i}=0,
$$

and

$$
D_{i} D_{i i} D_{j}=0, \quad D_{2} D_{11} D_{2}+D_{1} D_{22} D_{1}=0 .
$$

Proposition 2.7. Let $h=D_{11} D_{2} h_{12}+D_{22} D_{1} h_{21}$ be a generic element in $F_{2}$. Then $d^{2} h=0$ if and only if

$$
\partial_{1} h_{21}+\partial_{2} h_{12}=0
$$

i.e. $d^{2} h=0$ if and only if $h=\left(h_{12}, h_{21}\right)$ satisfies the compatibility condition for the system (5).

On the basis of what we know from the syzygies of the Dirac complex in two operators (see for example [3]) we would now expect that the complex of differential forms should naturally close to zero. In fact, in [5] we establish that the differential operator $d^{3}$ is identically zero on $F_{3}$ and therefore we have the following result that concludes the description in the case of two variables:

Theorem 2.8. Monogenic functions in two variables can be embedded in the following de Rhamlike complex:

$$
0 \rightarrow \mathcal{R} \hookrightarrow F_{0} \stackrel{d^{0}}{\rightarrow} F_{1} \stackrel{d^{1}}{\rightarrow} F_{2} \stackrel{d^{2}}{\rightarrow} F_{3} \stackrel{d^{3}}{\rightarrow} 0 .
$$

Remark 2.9. The de Rham-like complex which we have constructed is self dual in the sense that $d^{2}$ is the transpose of $d^{0}$. This is not surprising because the same structure occurs in the resolution of the Dirac system in two variables

$$
0 \longrightarrow R^{2^{m}}(-4) \longrightarrow R^{2 \cdot 2^{m}}(-3) \longrightarrow R^{2 \cdot 2^{m}}(-1) \longrightarrow R^{2^{m}} \longrightarrow \mathcal{M}_{2} \longrightarrow 0 .
$$

Remark 2.10. In [5] we have shown how to apply the same ideas to the case of three Dirac operators. Unfortunately the results are not yet complete, and so, in this paper, we have decided to limit our attention to the case of two operators. 
Remark 2.11. Note that the sequence we have constructed is in fact a fine resolution of the sheaf of monogenic functions. This is a consequence of the fact that each space $F_{j}$ can be thought of as a direct sum of a certain number of copies of the sheaf of infinitely differentiable functions, which in itself is a fine sheaf. This is an important remark, as fine resolutions are helpful in computing the cohomology of the sheaves they resolve. This fact is the starting point for [2].

\section{Quaternionic megaforms for the two dimensional Cauchy-Fueter system}

In this section we use the same ideas introduced in the case of Dirac systems to discuss megaforms for the two-dimensional Cauchy-Fueter system. We denote by $\mathbb{H}$ the algebra of quaternions and by $q=x_{0}+\mathbf{i} x_{1}+\mathbf{j} x_{2}+\mathbf{k} x_{3}$ a quaternion, where $x_{\ell} \in \mathbb{R}$ for $\ell=0, \ldots, 3$. We define the CauchyFueter operator as

$$
\frac{\partial}{\partial \bar{q}}=\frac{\partial}{\partial x_{0}}+\mathbf{i} \frac{\partial}{\partial x_{1}}+\mathbf{j} \frac{\partial}{\partial x_{2}}+\mathbf{k} \frac{\partial}{\partial x_{3}}
$$

with obvious meaning of the symbols. Differentiable functions which belong to the kernel of $\partial / \partial \bar{q}$ are called regular functions.

When we consider several quaternionic variables $q_{r}$, for $r=1, \ldots, n$, we can define a CauchyFueter operator for each of them by setting

$$
\frac{\partial}{\partial \overline{q_{r}}}=\frac{\partial}{\partial x_{r 0}}+\mathbf{i} \frac{\partial}{\partial x_{r 1}}+\mathbf{j} \frac{\partial}{\partial x_{r 2}}+\mathbf{k} \frac{\partial}{\partial x_{r 3}}
$$

where the double index for the variables has obvious meaning. Then, a differentiable function $f: \mathbb{H}^{n} \rightarrow \mathbb{H}$ is said to be regular if it is regular separately in each of its $n$ quaternionic variables. There are substantial, and important, differences between the Dirac case and the Cauchy-Fueter case; nevertheless, as indicated in [3], the case of dimension two still presents more similarities than differences, and so the techniques used in the previous section can be suitably modified for the quaternionic case. Again, as in the Dirac case, we introduce new symbols that will act as "differential forms". We construct the associative algebra generated by the degree one quaternionic megaforms

$$
\left\{\check{D}_{i}^{k}, D_{i}^{k} \mid i=1 \ldots n, k \in \mathbb{N}\right\}
$$

together with the degree two megaforms

$$
\left\{\check{D}_{i j}^{k}, D_{i j}^{k}, \tilde{D}_{i j}^{k}, D_{i j}^{* k} \mid i, j=1 \ldots n, k \in \mathbb{N}\right\} .
$$

Note that, as remarked before, we will omit the superscript $k$. As coefficients of the quaternionic megaforms we use the quaternionic derivatives $\partial_{\bar{q}_{i}}, i=1 \ldots n$ and their conjugates $\partial_{q_{i}}$. Together they generate an algebra and satisfy the relations

$$
\Delta_{j} \partial_{\bar{q}_{i}}=\partial_{\bar{q}_{i}} \Delta_{j}, \quad i, j=1 \ldots n
$$

and

$$
\Delta_{j} \partial_{q_{i}}=\partial_{q_{i}} \Delta_{j}, \quad i, j=1 \ldots n
$$

where the Laplacian symbol is defined as $\Delta_{j}=\partial_{\bar{q}_{j}} \partial_{q_{j}}=\partial_{q_{j}} \partial_{\bar{q}_{j}}$. The proofs for the results in this section can be found in [2], but we will include a couple of the simplest computations to convey the flavor of this approach. Let $\mathcal{R}$ be the space of regular functions in two variables $q_{1}$, 
$q_{2}$. Let $F_{0}$ be the space of $\mathcal{C}^{\infty}$ functions in $q_{1}, q_{2}$ and let $F_{1}$ be the space of "1-forms" whose elements are written as

$$
g=\sum_{i=1}^{2} \check{D}_{i} \check{g}_{i}, \quad \check{g}_{i} \in F_{0}, \quad i=1,2
$$

so that if $d^{0}=\sum_{i=1}^{2} \check{D}_{i} \bar{\partial}_{i}$, where $\bar{\partial}_{i}=\partial_{\bar{q}_{i}}$, we have an exact sequence

$$
0 \rightarrow \mathcal{R} \hookrightarrow F_{0} \stackrel{d^{0}}{\rightarrow} F_{1} .
$$

As before, we want to define a space $F_{2}$ of "2-forms" and a suitable "differential" $d^{1}: F_{1} \rightarrow F_{2}$ such that $d^{1} d^{0}=0$, and as before we assume that $d^{1}$ is made of two components $d_{1}^{1}$ and $d_{2}^{1}$ of degrees, respectively, one and two. Let us define

$$
d_{1}^{1}=\check{D}_{1} \bar{\partial}_{1}+\check{D}_{2} \bar{\partial}_{2}+D_{1} \partial_{1}+D_{2} \partial_{2}
$$

where $\partial_{i}$ denotes $\partial / \partial q_{i}$ and

$$
d_{2}^{1}=\sum_{i, j=1}^{2}\left(\check{D}_{i j} \bar{\partial}_{i} \bar{\partial}_{j}+D_{i j} \partial_{i} \partial_{j}+\tilde{D}_{i j} \partial_{i} \bar{\partial}_{j}+D_{i j}^{*} \bar{\partial}_{i} \partial_{j}\right)
$$

Remark 3.1. The condition $d^{1} d^{0}=0$ implies that $d_{1}^{1} d^{0}=0$, i.e.

$$
\sum_{j, k=1}^{2}\left(\check{D}_{k} \check{D}_{j} \bar{\partial}_{k} \bar{\partial}_{j}+D_{k} \check{D}_{j} \partial_{k} \bar{\partial}_{j}\right) f=0
$$

As a consequence, for any $k$ and $j, \check{D}_{k} \check{D}_{j}=0$ and $D_{k} \check{D}_{j}=0$. So one can assume that $d_{1}^{1} \equiv 0$, and therefore

$$
d^{1} g=d_{2}^{1} g .
$$

Proposition 3.2. Let $d^{0}=\check{D}_{1} \bar{\partial}_{1}+\check{D}_{2} \bar{\partial}_{2}$ and

$$
d^{1}=\sum_{i, j=1}^{2}\left(\check{D}_{i j} \bar{\partial}_{i} \bar{\partial}_{j}+D_{i j} \partial_{i} \partial_{j}+\tilde{D}_{i j} \partial_{i} \bar{\partial}_{j}+D_{i j}^{*} \bar{\partial}_{i} \partial_{j}\right)
$$

Then $d^{1} d^{0}=0$ implies the following:

$$
\begin{aligned}
& \left(\tilde{D}_{i i}+D_{i i}^{*}\right) \check{D}_{i}=0, \quad i=1,2, \\
& D_{j i}^{*} \check{D}_{i}+\left(\tilde{D}_{i i}+D_{i i}^{*}\right) \check{D}_{j}=0, \quad i, j=1,2, \quad i \neq j, \\
& D_{i j} \check{D}_{k}=0, \quad i, j, k=1,2, \\
& \check{D}_{i j} \check{D}_{k}=0, \quad i, j, k=1,2, \\
& \tilde{D}_{i j} \check{D}_{k}=0, \quad i, j, k=1,2, \quad i \neq j, \\
& D_{i j}^{*} \check{D}_{i}=0, \quad i, j=1,2, \quad i \neq j .
\end{aligned}
$$

Proof. The condition $d_{2}^{1} d^{0}=0$ implies that for any $f \in F_{0}$ it is

$$
\sum_{i, j=1}^{2}\left(\check{D}_{i j} \check{D}_{k} \bar{\partial}_{i} \bar{\partial}_{j} \bar{\partial}_{k}+D_{i j} \check{D}_{k} \partial_{i} \partial_{j} \bar{\partial}_{k}+\tilde{D}_{i j} \check{D}_{k} \partial_{i} \bar{\partial}_{j} \bar{\partial}_{k}+D_{i j}^{*} \check{D}_{k} \bar{\partial}_{i} \partial_{j} \bar{\partial}_{k}\right) f=0
$$

By writing explicitly the right hand term, and using the relation $\partial_{i} \bar{\partial}_{i} \bar{\partial}_{j}=\bar{\partial}_{j} \partial_{i} \bar{\partial}_{i}$, i.e. $\Delta_{i} \bar{\partial}_{j}=$ $\bar{\partial}_{j} \Delta_{i}, i, j=1,2$ where $\Delta_{i}$ denotes the Laplacian with respect to the $i$ th variable, and grouping the various terms we get the statement. 
We now consider the kernel of the map $d^{1}: F_{1} \rightarrow F_{2}$. We have that a 1 -form $g$ is $d^{1}$-closed if and only if its components $g_{j}$ satisfy the compatibility conditions of the system $d^{0} f=g$.

Proposition 3.3. Let $g=\check{D}_{1} g_{1}+\check{D}_{2} g_{2}$ be an element of $F_{1}$. Then $d^{1} g=0$ if and only if

$$
\bar{\partial}_{i} \partial_{i} g_{j}-\bar{\partial}_{j} \partial_{i} g_{i}=0, \quad i, j=1,2, i \neq j,
$$

i.e., $d^{1} g=0$ if and only if $\left(g_{1}, g_{2}\right)$ satisfy the compatibility conditions for the solvability of the system

$$
\left\{\begin{array}{l}
\bar{\partial}_{1} f=g_{1} \\
\bar{\partial}_{2} f=g_{2}
\end{array}\right.
$$

Proof. By the definition of $g$ and $d^{1}$ we have that $d^{1} g=0$ can be written as

$$
\begin{aligned}
& \check{D}_{11} \check{D}_{1} \bar{\partial}_{1}^{2} g_{1}+\check{D}_{12} \check{D}_{1} \bar{\partial}_{1} \bar{\partial}_{2} g_{1}+\check{D}_{21} \check{D}_{1} \bar{\partial}_{2} \bar{\partial}_{1} g_{1}+\check{D}_{22} \check{D}_{1} \bar{\partial}_{2}^{2} g_{1} \\
+ & D_{11} \check{D}_{1} \partial_{1}^{2} g_{1}+D_{12} \check{D}_{1} \partial_{1} \partial_{2} g_{1}+D_{21} \check{D}_{1} \partial_{2} \partial_{1} g_{1}+D_{22} \check{D}_{1} \partial_{2}^{2} g_{1} \\
+ & \tilde{D}_{11} \check{D}_{2} \partial_{1}^{2} g_{2}+\tilde{D}_{12} \check{D}_{2} \partial_{1} \partial_{2} g_{2}+\tilde{D}_{21} \check{D}_{2} \partial_{2} \partial_{1} g_{2}+\tilde{D}_{22} \check{D}_{2} \partial_{2}^{2} g_{2} \\
+ & D_{11} \check{D}_{2} \partial_{1}^{2} g_{2}+D_{12} \check{D}_{2} \partial_{1} \partial_{2} g_{2}+D_{21} \check{D}_{2} \partial_{2} \partial_{1} g_{2}+D_{22} \check{D}_{2} \partial_{2}^{2} g_{2}=0
\end{aligned}
$$

In view of (6) this can be rewritten as

$$
\begin{gathered}
D_{11}^{*} \check{D}_{1}\left(\bar{\partial}_{1} \partial_{1} g_{1}-\partial_{1} \bar{\partial}_{1} g_{1}\right)+D_{22}^{*} \check{D}_{2}\left(\bar{\partial}_{2} \partial_{2} g_{2}-\partial_{2} \bar{\partial}_{2} g_{2}\right) \\
+D_{22}^{*} \check{D}_{1}\left(\bar{\partial}_{2} \partial_{2} g_{1}-\bar{\partial}_{1} \partial_{2} g_{2}\right)+\tilde{D}_{22} \check{D}_{1}\left(\partial_{2} \bar{\partial}_{2} g_{1}-\bar{\partial}_{1} \partial_{2} g_{2}\right) \\
+D_{11}^{*} \check{D}_{2}\left(\bar{\partial}_{1} \partial_{1} g_{2}-\bar{\partial}_{2} \partial_{1} g_{1}\right)+\tilde{D}_{11} \check{D}_{2}\left(\partial_{1} \bar{\partial}_{1} g_{2}-\bar{\partial}_{2} \partial_{1} g_{1}\right)=0
\end{gathered}
$$

which completes the proof.

From the general theory, it is known that the complex closes with one more linear condition that is the compatibility condition for the solvability of the system

$$
\left\{\begin{array}{c}
\Delta_{1} g_{2}-\bar{\partial}_{2} \partial_{1} g_{1}=h_{12} \\
\Delta_{2} g_{1}-\bar{\partial}_{1} \partial_{2} g_{2}=h_{21}
\end{array}\right.
$$

This condition can be derived by simply using megaforms and their closure.

Proposition 3.4. Let

$$
d^{1}=\sum_{i, j=1}^{2}\left(\check{D}_{i j} \bar{\partial}_{i} \bar{\partial}_{j}+D_{i j} \partial_{i} \partial_{j}+\tilde{D}_{i j} \partial_{i} \bar{\partial}_{j}+D_{i j}^{*} \bar{\partial}_{i} \partial_{j}\right)
$$

and let

$$
\begin{gathered}
d^{2}=d_{1}^{2}+d_{2}^{2}=\check{D}_{1} \bar{\partial}_{1}+\check{D}_{2} \bar{\partial}_{2}+D_{1} \partial_{1}+D_{2} \partial_{2} \\
+\sum_{i, j=1}^{2}\left(\check{D}_{i j} \bar{\partial}_{i} \bar{\partial}_{j}+D_{i j} \partial_{i} \partial_{j}+\tilde{D}_{i j} \partial_{i} \bar{\partial}_{j}+D_{i j}^{*} \bar{\partial}_{i} \partial_{j}\right)
\end{gathered}
$$

then $d^{2} d^{1}=0$ implies for $i, j=1,2, i \neq j$

$$
\begin{gathered}
\check{D}_{1} D_{11}^{*} \check{D}_{2}+\check{D}_{1} \tilde{D}_{11} \check{D}_{2}=0, \\
\check{D}_{i} D_{j j}^{*} \check{D}_{i}+\check{D}_{i} \tilde{D}_{j j} \check{D}_{i}=0,
\end{gathered}
$$




$$
\begin{gathered}
\left(D_{1} D_{22}^{*} \check{D}_{1}+D_{1} \tilde{D}_{22} \check{D}_{1}\right)-\left(D_{2} D_{11}^{*} \check{D}_{2}+D_{2} \tilde{D}_{11} \check{D}_{2}\right)=0, \\
D_{i} D_{i i}^{*} \check{D}_{j}+D_{i} \tilde{D}_{i i} \check{D}_{j}=0, \\
D_{2} D_{22}^{*} \check{D}_{1}+D_{2} \tilde{D}_{22} \check{D}_{1}=0, \\
\tilde{D}_{i i} D_{i i}^{*} \check{D}_{j}+\tilde{D}_{i i} \tilde{D}_{i i} \check{D}_{j}+D_{i i}^{*} D_{i i}^{*} \check{D}_{j}+D_{i i}^{*} \tilde{D}_{i i} \check{D}_{j}=0, \quad i, j=1,2, \quad i \neq j, \\
D_{i i} D_{j j}^{*} \check{D}_{i}+D_{i i} \tilde{D}_{j j} \check{D}_{i}-D_{i j} D_{i i}^{*} \check{D}_{j}-D_{i j} \tilde{D}_{i i} \check{D}_{j}=0, \quad i, j=1,2, \quad i \neq j, \\
\tilde{D}_{i i} D_{j j}^{*} \check{D}_{i}+\tilde{D}_{i i} \tilde{D}_{j j} \check{D}_{i}+D_{i i}^{*} D_{j j}^{*} \check{D}_{i}+D_{i i}^{*} \tilde{D}_{j j} \check{D}_{i}-D_{i j}^{*} D_{i i}^{*} \check{D}_{j}-D_{i j}^{*} \tilde{D}_{i i} \check{D}_{j}=0, \quad i, j=1,2, \quad i \neq j, \\
\check{D}_{i j} D_{k k}^{*} \check{D}_{\ell}+\check{D}_{i j} \tilde{D}_{k k} \check{D}_{\ell}=0, \quad i, j, k, \ell=1,2, \quad k \neq \ell, \quad \\
D_{i i} D_{i i}^{*} \check{D}_{j}+D_{i i} \tilde{D}_{i i} \check{D}_{j}=0, \quad i, j,=1,2, \quad i \neq j, \\
\tilde{D}_{i j} D_{k k}^{*} \check{D}_{\ell}+\tilde{D}_{i j} \tilde{D}_{k k} \check{D}_{\ell}=0, \quad i, j, k, \ell=1,2, \quad k \neq \ell, \quad i \neq j, \\
D_{j i}^{*} D_{i i}^{*} \check{D}_{j}+D_{j i}^{*} \tilde{D}_{i i} \check{D}_{j}=0, \quad i, j,=1,2, \quad i \neq j, \\
D_{j i} D_{i i}^{*} \check{D}_{j}+D_{j i} \tilde{D}_{i i} \check{D}_{j}=0, \quad i, j,=1,2, \quad i \neq j .
\end{gathered}
$$

Proposition 3.5. Let

$$
h=\left(D_{11}^{*} \check{D}_{2}+\tilde{D}_{11} \check{D}_{2}\right) h_{12}+\left(D_{22}^{*} \check{D}_{1}+\tilde{D}_{22} \check{D}_{1}\right) h_{21}
$$

be a generic element in $F_{2}$. Then $d^{2} h=0$ if and only if

$$
\partial_{1} h_{21}+\partial_{2} h_{12}=0
$$

i.e. $d^{2} h=0$ if and only if $h=\left(h_{12}, h_{21}\right)$ satisfies the compatibility condition for the system (8).

The analysis can be pushed one more step, to the closure of the complex, as indicated in the next two propositions from [2].

Proposition 3.6. Let

$$
\begin{gathered}
d^{3}=d_{1}^{3}+d_{2}^{3}=\check{D}_{1} \bar{\partial}_{1}+\check{D}_{2} \bar{\partial}_{2}+D_{1} \partial_{1}+D_{2} \partial_{2} \\
+\sum_{i, j=1}^{2}\left(\check{D}_{i j} \bar{\partial}_{i} \bar{\partial}_{j}+D_{i j} \partial_{i} \partial_{j}+\tilde{D}_{i j} \partial_{i} \bar{\partial}_{j}+D_{i j}^{*} \bar{\partial}_{i} \partial_{j}\right)
\end{gathered}
$$

Then $d^{3} d_{1}^{2}=0$ implies

(1) $\check{D}_{i} D_{1} D_{22}^{*} \check{D}_{1}+\check{D}_{i} D_{1} \tilde{D}_{22} \check{D}_{1}=0, \quad i=1,2$,

(2) $D_{i} D_{1} D_{22}^{*} \check{D}_{1}+D_{i} D_{1} \tilde{D}_{22} \check{D}_{1}=0, \quad i=1,2$,

(3) $\left(\tilde{D}_{i i} D_{1} D_{22}^{*} \check{D}_{1}+\tilde{D}_{i i} D_{1} \tilde{D}_{22} \check{D}_{1}\right)+\left(D_{i i}^{*} D_{1} D_{22}^{*} \check{D}_{1}+D_{i i}^{*} D_{1} \tilde{D}_{22} \check{D}_{1}\right)=0, \quad i, j=1,2, i \neq j$,

(4) $\left(D_{i j} D_{1} D_{22}^{*} \check{D}_{1}+D_{i j} D_{1} \tilde{D}_{22} \check{D}_{1}\right)=0, \quad i, j=1,2$,

(5) $\left(\check{D}_{i j} D_{1} D_{22}^{*} \check{D}_{1}+\check{D}_{i j} D_{1} \tilde{D}_{22} \check{D}_{1}\right)=0, \quad i, j=1,2$,

(6) $\left(\tilde{D}_{i j} D_{1} D_{22}^{*} \check{D}_{1}+\tilde{D}_{i j} D_{1} \tilde{D}_{22} \check{D}_{1}\right)=0, \quad i, j=1,2, i \neq j$,

(7) $\left(D_{i j}^{*} D_{1} D_{22}^{*} \check{D}_{1}+D_{i j}^{*} D_{1} \tilde{D}_{22} \check{D}_{1}\right)=0, \quad i, j=1,2, i \neq j$. 
Proposition 3.7. Let

$$
k=\left(D_{1} D_{22}^{*} \check{D}_{1}+D_{1} \tilde{D}_{22} \check{D}_{1}\right) k_{0}
$$

be a generic element in $F_{3}$. Then $d^{3} k=0$.

The interest of these results is clarified by the next theorem and remark.

Theorem 3.8. Regular functions in two quaternionic variables can be embedded in the following Dolbeault-like complex:

$$
0 \rightarrow \mathcal{R} \hookrightarrow F_{0} \stackrel{d^{0}}{\rightarrow} F_{1} \stackrel{d^{1}}{\rightarrow} F_{2} \stackrel{d^{2}}{\rightarrow} F_{3} \stackrel{d^{3}}{\rightarrow} 0 .
$$

Remark 3.9. If we denote by $\mathcal{C}^{\infty}$ the sheaf of quaternionic valued infinitely differentiable functions on $\mathbb{H}^{2}$, we can rewrite the various spaces of megaforms as follows:

$$
\begin{aligned}
& F_{0}:=\mathcal{C}^{\infty}, \\
& F_{1}:=\left\{\sum_{i=1}^{2} \check{D}_{i} \check{g}_{i}, \quad \check{g}_{i} \in \mathcal{C}^{\infty}\right\}, \\
& F_{2}:=\left\{\left(D_{11}^{*} \check{D}_{2}+\tilde{D}_{11} \check{D}_{2}\right) h_{12}+\left(D_{22}^{*} \check{D}_{1}+\tilde{D}_{22} \check{D}_{1}\right) h_{21}: h_{12}, h_{21} \in \mathcal{C}^{\infty}\right\}, \\
& F_{3}:=\left\{\left(D_{1} D_{22}^{*} \check{D}_{1}+D_{1} \tilde{D}_{22} \check{D}_{1}\right) k_{0}: k_{0} \in \mathcal{C}^{\infty}\right\} .
\end{aligned}
$$

This shows, in particular, that we have in fact constructed a fine resolution for the sheaf of regular functions in two variables.

\section{Applications of megaforms, and further directions for research}

The previous two sections have shown that, at least when we restrict our attention to the case of two operators, it is possible to embed the sheaves $\mathcal{M}$ and $\mathcal{R}$ in suitable fine resolutions whose objects are sheaves of differential forms (actually, what we have called megaforms) with infinitely differentiable coefficients.

These embeddings are very important as they allow us to calculate explicitly the cohomology of the sheaves of regular and monogenic functions. This, in turn, will allow the construction of suitable sheaves of hyperfunctions (see [3] for a quick review of related notions, and their interpretation in the quaternionic and Clifford settings). Indeed, classical hyperfunctions are constructed as a derived sheaf of the sheaf of holomorphic functions. For this process to work when the sheaf of holomorphic functions is replaced by a different sheaf, one needs some properties on the vanishing of suitable cohomologies. This vanishing is provided by the introduction of megaforms.

Let us therefore mention here some of the most important results which we have discussed and fully proved in [2]. To begin with, the usual arguments using fine sheaves prove the following result:

Theorem 4.1. Let $U$ be an open convex set in $\mathbb{H}^{2}$. Then, for any $j \geq 1$, it is

$$
H^{j}(U, \mathcal{R})=0 .
$$

Its monogenic version states:

Theorem 4.2. Let $U$ be an open convex set in $\mathbb{R}^{2 m}$. Then, for any $j \geq 1$, it is

$$
H^{j}(U, \mathcal{M})=0 .
$$


These results allow us to introduce suitable spaces of hyperfunctions.

Definition 4.3. Let $L$ be an initial variety for the Cauchy-Fueter system in two variables (see [2] for details). The presheaf associated to the pairing $\left(V, H_{L}^{3}(V, \mathcal{R})\right)$, with $V$ an open set in $L$, is a sheaf which we will call sheaf of $\mathbb{H}$-hyperfunctions. In the sequel we will denote this sheaf by $\mathcal{B}_{\mathbb{H}^{2}, L}$.

The vanishing properties of the cohomology groups of $\mathcal{R}$ and the definition above imply the following:

Theorem 4.4. Let $U$ be any open convex set in $\mathbb{H}^{2}$ and let $L$ be a five-dimensional initial variety for the Cauchy-Fueter system. Then $\mathcal{B}_{\mathbb{H}^{2}, L}(U \cap L) \cong H^{2}(U \backslash L, \mathcal{R})$.

Note now that the open set $U \backslash L$ is not convex, and therefore its $\mathcal{R}$ cohomology does not vanish. In fact, our results show that its elements are equivalence classes of two-forms so that we can identify any quaternionic hyperfunction with an equivalence class of two-forms. By recalling the characterization of $F_{2}$ and $F_{1}$, we can identify a quaternionic hyperfunction $f$ on $U \cap L$ with an equivalence class of pairs $\left(f_{1}, f_{2}\right)$ of quaternionic valued, infinitely differentiable functions on $U \backslash L$, such that $\partial_{1} f_{1}+\partial_{2} f_{2}=0$. Two pairs $\left(f_{1}, f_{2}\right)$ and $\left(g_{1}, g_{2}\right)$ are equivalent if there exist another pair $\left(\alpha_{1}, \alpha_{2}\right)$ such that

$$
\left\{\begin{array}{l}
\Delta_{1} \alpha_{2}-\bar{\partial}_{2} \partial_{1} \alpha_{1}=f_{1}-g_{1} \\
\Delta_{2} \alpha_{1}-\bar{\partial}_{1} \partial_{2} \alpha_{2}=f_{2}-g_{2}
\end{array}\right.
$$

One can proceed in a totally analogous way for the case of monogenic functions.

Definition 4.5. Let $L$ be an initial variety for the Dirac system in two variables. The presheaf associated to the pairing $\left(V, H_{L}^{3}(V, \mathcal{M})\right)$, with $V$ an open set in $L$, is a sheaf which we will call sheaf of Dirac-hyperfunctions. In the sequel we will denote this sheaf by $\mathcal{D}_{\mathbb{R}^{2 m}, L}$.

Using the fine resolution we have described for the sheaf of monogenic functions we can deduce the Clifford analogues of all the previous results. The cohomology of $\mathcal{M}$ vanishes on all convex open sets in $\mathbb{R}^{2 m}$, and the sheaf of hyperfunctions ends up being isomorphic to an appropriate sheaf of 2-megaforms.

There are still many interesting open problems. For example, one would like to extend these arguments to the case of more than two variables. In [5] we have shown how to construct a theory of megaforms for the Dirac operators. However, the theory we have constructed is not fully complete, and is not sufficient to recuperate all the results announced in this paper. The case of Dirac operators seems to be independent of the number of operators, but we would like to be able to prove this conclusively, so to have a general theory of Dirac-hyperfunctions, irrespective of dimension.

The situation is much more complicated in the case of quaternions. As we have shown, the case of three Cauchy-Fueter operators is quite distinctive, and introduces new phenomena which did not occur in dimension two. We do not have, at this time, an appropriate megaform theory for this case, nor we have a clear idea on how this could be done. On the positive side, we know that no new phenomena emerge when we consider more than three Cauchy-Fueter operators, so that if we are able to understand the case of three operators, we should be able to have a general theory.

Finally, and more ambitiously, we believe it should be possible to construct a general theory of abstract megaforms for general systems of differential equations. Since such a theory would imply some vanishing of cohomologies, we should be prepared to find conditions on a system 
for it to have an associated theory of megaforms (in other words, we can envision differential operators, whose kernels have non-vanishing cohomology, and for these operators we could not construct appropriate megaforms). The development of such a theory would rely on an appropriate understanding of the syzygies of the operators, and one should be open to the case in which higher order megaforms may be needed. We hope to return to all these questions in the not too far future.

\section{References}

[1] F. Brackx, R. Delanghe, F. Sommen, Clifford Analysis, Pitman Res. Notes in Math., 76, 1982.

[2] F. Colombo, A. Damiano, I. Sabadini, D. C. Struppa, Quaternionic hyperfunctions on 5-dimensional varieties in $\mathbb{H}^{2}$, Politecnico di Milano, preprint, 2005.

[3] F. Colombo, I. Sabadini, F. Sommen, D. C. Struppa, Analysis of Dirac Systems and Computational Algebra, Progress in Mathematical Physics, Birkhäuser Verlag, 2004.

[4] J. Gilbert, M. Murray, Clifford Algebras and Dirac Operators in Harmonic Analysis, Cambridge, Cambridge Univ. Press n. 26, 1990.

[5] I. Sabadini, F. Sommen, D.C. Struppa, The Dirac complex on abstract vector variables: megaforms, Exp. Math., 12 (3) (2003), 351-364.

[6] F. Sommen, Microfunctions with values in a Clifford algebra. II, Sci. Papers College Arts Sci. Univ. Tokyo, 36 (1) (1986), 15-37. 